\title{
Isolated and early-onset cerebral fat embolism syndrome in a multiply injured patient: a rare case
}

\author{
Chin-Kai Huang ${ }^{1}$, Chih-Yuan Huang ${ }^{2,3}$, Chia-Lung Li ${ }^{1,3}$, Jui-Ming Yang ${ }^{4,5}$, Chin-Hsien Wu ${ }^{6}$, Chih-Hui Chen ${ }^{7,8^{*}}$ and \\ Po-Ting $\mathrm{Wu}^{1,3,5,9,10^{*}}$
}

\begin{abstract}
Background: Fat embolism syndrome (FES) is a rare complication that can occur between 12 and $72 \mathrm{~h}$ after the initial insult. Isolated cerebral FES without pulmonary symptoms is rarer. Early fracture fixation might prevent FES. We report a case of multiple-fracture with FES despite definite fixation three hours post-injury.

Case presentation: A 54-year-old man presented with multiple fractures: left femoral shaft (AO B2), left distal radius (AO C3), left comminuted patella, right comminuted 1st metatarsal base and left 2nd-4th metatarsal neck. Because he was stable, we gave him early total care and definite fixation, which required seven hours and yielded no complications. After he recovered from anesthesia, however, his eyes deviated right, his right upper arm was paralyzed, his consciousness level was poor, and his Glasgow Coma Scale score was E3VeM4. Chest X-rays showed clear lung fields, and brain computed tomography showed no intracranial hemorrhage. He did, however, have tachycardia, anemia, and thrombocytopenia. Brain magnetic resonance images showed a hyperintensive starfield pattern on diffuse weighted images, which suggested cerebral FES. After supportive care, his consciousness cleared on postoperative day 17, and he recovered full right upper arm muscle power after four months; however, he had a significant cognitive deficit. One-year post-injury, after regular rehabilitation therapy, he was able to independently perform his activities of daily living but still had a residual mild cognitive deficit.
\end{abstract}

Conclusion: Early fixation can attenuate but not eliminate the incidence of FES. Early assessment and rehabilitation therapy might be required for patients with cerebral FES and cognitive deficits; however, such deficits are difficult to predict and need long-term follow-ups.

Keywords: Cerebral fat embolism syndrome, Cognitive deficit, Long-bone fracture, Outcome

\section{Background}

Fat embolism syndrome (FES) is a rare complication with a triad of symptoms: progressive respiratory distress, deteriorating mental status, and petechial rash. FES occurs between 12 and $72 \mathrm{~h}$ after the initial insult, and, in Taiwan, at a mean of $48.5 \mathrm{~h}$ after long-bone fractures [1]. According to the literature [2], the incidence ranges from $<1 \%$ to $>30 \%$ of cases. This wide variation in the incidence rate might be attributable to heterogeneous diagnosis criteria.

\footnotetext{
* Correspondence: orth.chen@gmail.com; anotherme500@gmail.com 7 Department of Orthopedics, Taichung Veterans General Hospital, 1650Boulevard Sect. 4, Taichung, Taiwan

'Department of Orthopedics, National Cheng Kung University Hospital, College of Medicine, National Cheng Kung University, Tainan, Taiwan Full list of author information is available at the end of the article
}

The most frequently used criteria are Gurd's [3] and Gurd and Wilson's [4], which require that a diagnosis of FES meet at least one major criterion and at least four minor criteria. Seventy-five percent of patients with FES had respiratory dysfunction because of tachypnea, hypoxemia, or respiratory failure [1], and $86 \%$ had cerebral changes [5]. However, a pure central nervous system presentation without pulmonary symptoms is rare [1]. We report a rare case with multiple fractures followed by isolated cerebral FES, despite the patient's having undergone early (threehours post-insult) total care with definite fixation.

(c) The Author(s). 2019 Open Access This article is distributed under the terms of the Creative Commons Attribution 4.0 International License (http://creativecommons.org/licenses/by/4.0/), which permits unrestricted use, distribution, and reproduction in any medium, provided you give appropriate credit to the original author(s) and the source, provide a link to the Creative Commons license, and indicate if changes were made. The Creative Commons Public Domain Dedication waiver (http://creativecommons.org/publicdomain/zero/1.0/) applies to the data made available in this article, unless otherwise stated. 


\section{Case report}

A 54-year-old man with no known underlying disease fell $4 \mathrm{~m}$ and was brought to the National Cheng Kung Hospital (NCKUH) emergency room. He had multiple fractures: left femoral shaft (AO B2), left distal radius (AO C3), left comminuted patella, right comminuted 1st metatarsal base (Fig. 1a-d), and left 2nd-4th metatarsal neck. A whole-body computed tomography (CT) scan showed no other associated injuries. About $3 \mathrm{~h}$ post-injury, he was sent to our operating room (OR) with a clear consciousness and $98-100 \%$ of peripheral oxygenation saturation in a normal indoor room atmosphere. Because he was physiologically stable, he was given early total care and a definite fracture fixation. Surgery lasted $7 \mathrm{~h}$ and yielded no complications. Closed reduction (CR) and internal fixation with a reamed antegrade interlocking nail for the left femur, open reduction and internal fixation with plates for the left distal radius and right 1st metatarsal base, and CR and fixation with Kirschner wires for the left 2nd-4th metatarsal neck were fixed in that order. Because the articular fracture of his left patella was unrepairable, interfragment Kirschner wires and patellotibial figure-of-eight wiring were used to restore the extensor mechanism. We found neither hypotension nor hypoxia during the surgery; postoperatively, however, the patient's eyes deviated to the right side, his right upper arm muscle was paralyzed, and his Glasgow Coma Scale (GCS) score was E3V $\mathrm{E}_{\mathrm{E}} \mathrm{M} 4$ when he recovered from the anesthesia. A chest X-ray (Fig. 2a) shows a clear lung field, and a brain CT (Fig. 2b) shows no intracranial hemorrhage. Despite the patient's low oxygen demand and no clinical evidence of acute respiratory distress syndrome $\left(\mathrm{FiO}_{2}: 30 \% ; \mathrm{PaO}_{2}: 108.6\right.$ $\mathrm{mmHg} ; \mathrm{FiO}_{2} / \mathrm{PaO}_{2}>300$ ), his endotracheal tube was retained for airway protection. On postoperative day (POD) 0 , the patient had tachycardia (heart rate: $110 / \mathrm{min}$ ), anemia (hemoglobin: $10.3 \mathrm{~g} / \mathrm{dL}$ ), and thrombocytopenia (platelet count: $131 \times 10^{3} / \mu \mathrm{L}$ ). He met only 1 major and 3 minor criteria. However, brain magnetic resonance image (MRI) (Fig. 2c) shows multiple nodular or punctate hyperintensity lesions on diffusion-weighted (DW) images of the bilateral thalami, basal ganglia, cerebellar, and cerebral hemispheres. We highly suspected FES and we gave the patient supportive treatment with additional methylprednisolone (Solu-Medrol ${ }^{\mathrm{m}}$ ) and albumin. His GCS score fell to $\mathrm{E} 2 \mathrm{~V}_{\mathrm{E}} \mathrm{M} 4$ on POD 4 and persisted at that level for the next 10 days. After POD 15, his consciousness improved and he was alert on POD 17, but his right upper arm muscle power score was 0 and left upper arm muscle power score was 3. On POD 29, his upper arm muscle power score was 3 on both sides. He could speak using only simple words instead of sentences, and he responded slowly. Four months post-injury, a complete cognitive test-the cognitive ability screening instrument (CASI) and the mini-mental status examination (MMSE)—showed significant deficits in recent memory, orientation, abstract

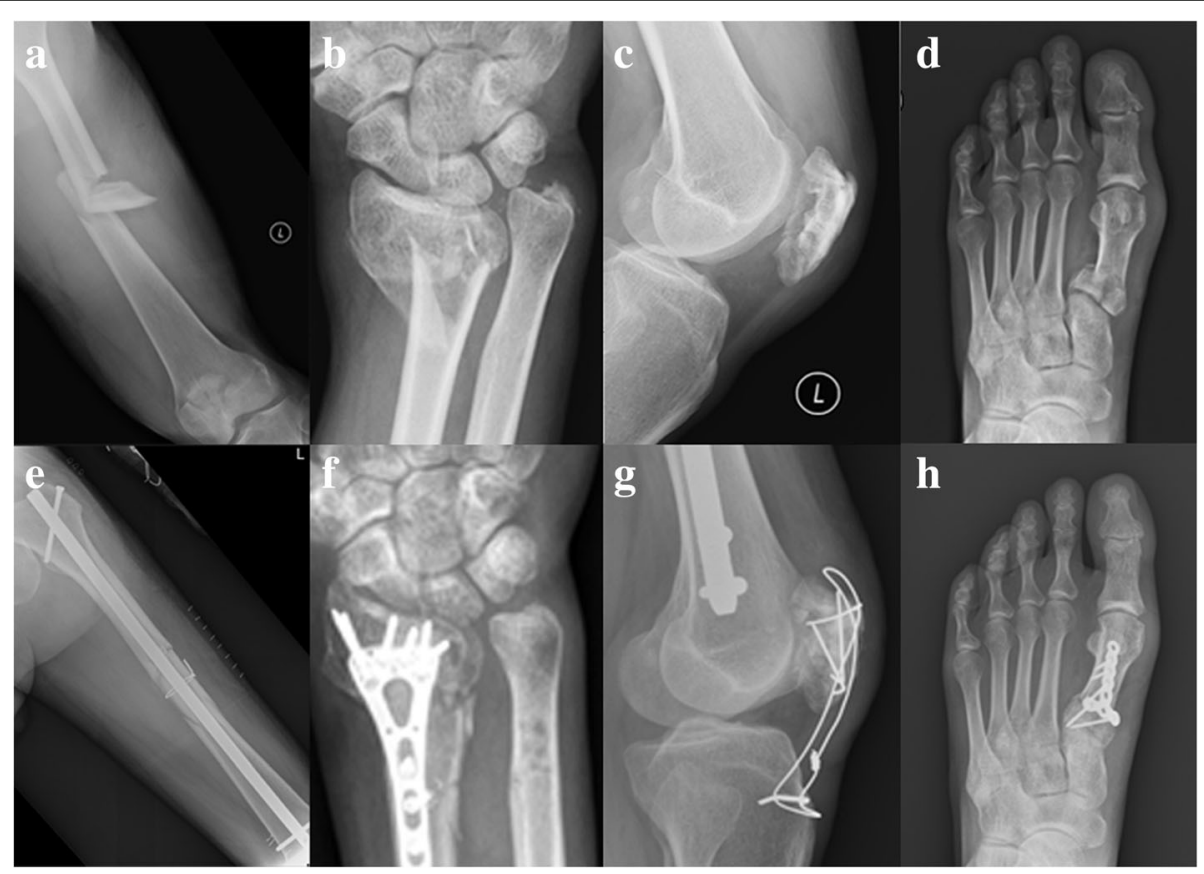

Fig. 1 Fractures before and after surgery. a Left femoral shaft (AO B2). b Left distal radius (AO C3). c Left comminuted patella. d Right comminuted 1st metatarsal base. e Closed reduction and internal fixation with a reamed antegrade interlocking nail for the left femur. $\mathbf{f}$ Open reduction and internal fixation with plates for the left distal radius. $\mathbf{g}$ Interfragment Kirschner wires and patellotibial figure-of-eight wiring for the left patella. $\mathbf{h}$ Open reduction and internal fixation with plates for the right 1st metatarsal base 

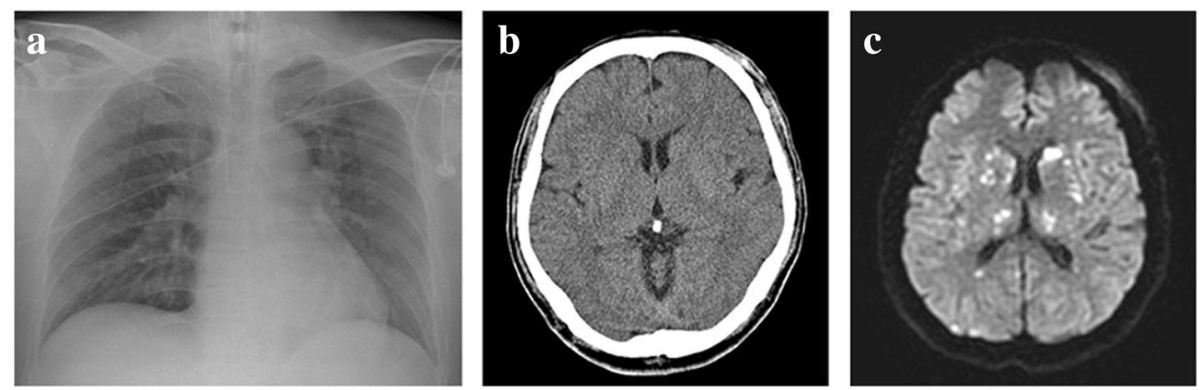

Fig. 2 a A chest X-ray of a clear lung field. b A brain CT showing no intracranial hemorrhage. c A brain MRI showing multiple nodular or punctate hyperintensity lesions on diffusion-weighted (DW) images of the bilateral thalami, basal ganglia, cerebellar, and cerebral hemispheres

thinking, and verbal fluency. The patient's right upper and lower limb muscle power had fully recovered, however. Because of his cognitive impairment and left limb muscle atrophy, he underwent physical and cognitive rehabilitation. All fractures achieved union four months post-surgery (Fig. 1e-h). One-year post-injury, the patient's left lower limb muscle power score had not fully recovered from left symptomatic traumatic patellofemoral joint arthritis. The muscle power scores of the other three limbs were full (five), and he was able to independently perform his activities of daily living. However, he continued to have a residual mild cognitive impairment.

\section{Discussion and conclusion}

As in most other case reports and series on FES [6], fat and medullary content flowed into our patient's bloodstream. However, FES has been reported in only 0.5$11 \%$ of patients with long-bone fractures [1], which might indicate that fat emboli are not significant for cardiopulmonary dysfunction. The stress response mediated by catecholamine is thought to be another possible mechanism of FES [7]. Isolated cerebral FES with primary neurological deficits is rare: however, an incidence of $10 \%$ was reported in one study [4]. Most FES cases have occurred between 12 and $72 \mathrm{~h}$ post-injury [1, 8], but one case of early-onset FES ( $<12 \mathrm{~h}$ post-insult) has been reported [9]. Patients with long-bone fractures and multiple fractures are at high risk for developing FES [2]. Early fixation can reduce the incidence of FES [10]. However, in our case, the patient underwent fixation $3 \mathrm{~h}$ post-injury but developed FES. Therefore, early fixation might attenuate but not eliminate FES.

The current diagnostic criteria of FES are based on clinical signs and symptoms; Gurd's and Gurd and Wilson's criteria are most frequently cited [2]. Our case met only one major criterion (conscious change) and three minor criteria (tachycardia, thrombocytopenia, and anemia), which does not fully support the diagnosis of FES. However, Gurd's [3] and Gurd and Wilson's [4] criteria were more favorable on pulmonary FES, and MRIs have shown their potential for diagnosing cerebral FES early. Multiple small nodular or hyperintense punctate lesions on the DW MRI, just like a starfield pattern of scattered bright spots on a dark background, is a typical finding for cerebral FES [11]. It is thought that scattered bright spots are pathognomonic of acute cerebral microinfarcts and that the hyperintense signal reflects foci of cytotoxic edema [11]. Therefore, researchers emphasize the importance of MRIs, and some even consider it a minor criterion of FES [12]. However, diffuse axonal injury (DAI) might also present the same starfield pattern on DW MRI even though most patients with DAI immediately present with an unclear consciousness after a head injury [13]. Our DW MRI finding was typical, and the clinical presentations met the rule of "one major criterion and four minor criteria" for diagnosing FES after we added the minor criterion of a DW MRI starfield pattern. Finally, we diagnosed our patient with cerebral FES after we had excluded other intracranial lesions.

The mortality of FES is about $5-15 \%$, and pulmonary dysfunction is its primary cause [1]. Although some devastating cases of massive cerebral FES have been reported [14-16], the prognosis of cerebral FES is generally favorable, with only small cognitive deficits or nearly full recovery in most cases [1]. Normal muscle tone with an active deep tendon reflex and retention of appropriate responses to pain are thought to be good prognostic signs [1]. However, cognitive impairment and mental disorders have not often been discussed in the literature. Two exceptions to this have reported a detailed neuropsychological assessment of one patient [17] and the long-term cognitive outcomes of another [18] with cerebral FES.

Cerebral manifestations of FES are highly variable and nonspecific. Mild cognitive dysfunction might be difficult to identify and is often underestimated because cerebral FES patients might have comorbid post-traumatic stress. Detailed cognitive and neurological assessments might help identify subtle cognitive dysfunction and might be indicated for all cerebral FES patients. Moreover, early diagnosis and early intervention might contribute to better outcomes $[19,20]$. Cerebral FES treatment is primarily 
supportive [21]. The importance of adequate oxygenation to prevent secondary brain injury is emphasized during recovery from cerebral FES [22].

We reported a rare case of early-onset and isolated cerebral FES in which the patient underwent surgery for his multiple fractures three hours post-trauma. Early fixation can attenuate but not eliminate the incidence of FES. MRIs have become more important for diagnosing FES. Better supportive care might lead to more favorable prognoses. Early cognitive assessment and rehabilitation therapy might be indicated for all cerebral FES patients with an apparent cognitive impairment; however, such impairments are difficult to predict and require longterm follow-ups.

\section{Abbreviations \\ CASI: Cognitive ability screening instrument; CR: Closed reduction; $C T$ : Computed tomography; DAl: Diffuse axonal injury.; DW: Diffusion- weighted; FES: Fat embolism syndrome; GCS: Glasgow Coma Scale; MMSE: Mini-Mental State Examination; MRI: Magnetic resonance image}

\section{Acknowledgements}

We are grateful to Skeleton Materials and Bio-compatibility Core Lab, Research Center of Clinical Medicine, National Cheng Kung University Hospital, and Yu-Ying Chen for the assistance of this study.

\section{Authors' contributions}

$\mathrm{CKH}, \mathrm{CYH}$, and PTW: study conception and design. CKH, CLL, JMY: data acquisition. $\mathrm{CKH}, \mathrm{CYH}, \mathrm{CHW}, \mathrm{CHC}$, and PTW: data analysis and interpretation. $\mathrm{CKH}, \mathrm{CYH}, \mathrm{CHC}$, and PTW: wrote the manuscript. $\mathrm{CYH}, \mathrm{CHC}$, and PTW: critical revision of the manuscript. All authors have read and approved the final submitted manuscript.

\section{Funding}

This work was supported by National Cheng Kung University Hospital (grants: NCKUH-10603023, NCKUH-10702024, and NCKUH-10809001). The funder had no role in study design, data collection and analysis, decision to publish, or preparation of the manuscript.

\section{Availability of data and materials}

All data concerning the case are presented in the manuscript.

\section{Ethics approval and consent to participate}

The study was approved by the NCKUH Institutional Review Board (IRB \#: BEC-108-008).

\section{Consent for publication}

Written, signed informed consent to publish this report and any accompanying images was obtained from the patient.

\section{Competing interests}

The authors declare that they have no competing interests.

\section{Author details}

${ }^{1}$ Department of Orthopedics, National Cheng Kung University Hospital, College of Medicine, National Cheng Kung University, Tainan, Taiwan. ${ }^{2}$ Department of Surgery, National Cheng Kung University Hospital, College of Medicine, National Cheng Kung University, Tainan, Taiwan. ${ }^{3}$ Division of Traumatology, Department of Surgery, National Cheng Kung University Hospital, College of Medicine, National Cheng Kung University, Tainan, Taiwan. ${ }^{4}$ Department of Orthopedics, Tainan ShinLau Christian Hospital, Tainan, Taiwan. ${ }^{5}$ Department of Biomedical Engineering, National Cheng Kung University, Tainan, Taiwan. ${ }^{6}$ Department of Orthopedics, E-Da Hospital/ I-Shou University, Kaohsiung, Taiwan. ${ }^{7}$ Department of Orthopedics, Taichung Veterans General Hospital, 1650Boulevard Sect. 4, Taichung, Taiwan. ${ }^{8}$ School of Medicine, National Yang-Ming University, Taipei, Taiwan. ${ }^{9}$ Department of Orthopedics, College of Medicine, National Cheng Kung University, 1
University Road, Tainan, Taiwan. ${ }^{10}$ Department of Orthopedics, National Cheng Kung University Hospital Dou-Liou branch, College of Medicine, National Cheng Kung University, YunLin, Taiwan.

Received: 3 November 2018 Accepted: 23 July 2019

Published online: 17 August 2019

\section{References}

1. Meyer N, Pennington WT, Dewitt D, Schmeling GJ. Isolated cerebral fat emboli syndrome in multiply injured patients: a review of three cases and the literature. J Trauma. 2007;63(6):1395-402.

2. Kosova E, Bergmark B, Piazza G. Fat embolism syndrome. Circulation. 2015; 131(3):317-20.

3. Gurd AR. Fat embolism: an aid to diagnosis. J Bone Joint Surg British Vol. 1970:52(4):732-7.

4. Gurd AR, Wilson RI. The fat embolism syndrome. J Bone Joint Surg British Vol. 1974;56b(3):408-16.

5. Kokatnur L, Rudrappa M, Khasawneh KR. Cerebral fat embolism: use of MR spectroscopy for accurate diagnosis. Ann Indian Acad Neurol. 2015;18(2): 252-5.

6. Gitin TA, Seidel T, Cera PJ, Glidewell OJ, Smith JL. Pulmonary microvascular fat: the significance? Crit Care Med. 1993;21(5):673-7.

7. Baker PL, Pazell JA, Peltier LF. Free fatty acids, catecholamines, and arterial hypoxia in patients with fat embolism. J Trauma. 1971;11(12):1026-30.

8. Tsai IT, Hsu CJ, Chen YH, Fong YC, Hsu HC, Tsai CH. Fat embolism syndrome in long bone fracture--clinical experience in a tertiary referral center in Taiwan. J Chin Med Assoc. 2010;73(8):407-10.

9. $\mathrm{Xu} \mathrm{XL}, \mathrm{Xu} \mathrm{P}$, Zheng $\mathrm{RQ}$, Lin $\mathrm{H}$, Chen $\mathrm{QH}$. A case of cerebral fat embolism. Chin Med J. 2016;129(14):1761-2.

10. Bone LB, Johnson KD, Weigelt J, Scheinberg R. Early versus delayed stabilization of femoral fractures: a prospective randomized study. 1989. Clin Orthop Relat Res. 2004;422:11-6.

11. Parizel PM, Demey HE, Veeckmans G, Verstreken F, Cras P, Jorens PG, et al, Early diagnosis of cerebral fat embolism syndrome by diffusion-weighted MRI (starfield pattern). Stroke. 2001;32(12):2942-4.

12. Eguia P, Medina A, Garcia-Monco JC, Martin V, Monton Fl. The value of diffusion-weighted MRI in the diagnosis of cerebral fat embolism. J Neuroimaging. 2007;17(1):78-80.

13. Su E, Bell M. Diffuse axonal injury. In: Laskowitz D, Grant G, editors, Translational research in traumatic brain injury. Boca Raton (FL: CRC Press/ Taylor and Francis Group; 2016.

14. Walshe CM, Cooper JD, Kossmann T, Hayes I, lles L. Cerebral fat embolism syndrome causing brain death after long-bone fractures and acetazolamide therapy. Crit Care Resusc. 2007;9(2):184-6.

15. Kawati R, Larsson A. Brain death due to fat embolism - could moderate hypercapnia and prone position be blamed for the tonsillar herniation? Ups J Med Sci. 2013;118(4):276-8.

16. Aggarwal R, Pal S, Soni KD, Gamangatti S. Massive cerebral fat embolism leading to brain death: a rare presentation. Indian J Crit Care Med. 2015; 19(11):687-9.

17. Gray AC, Torrens L, White TO, Carson A, Robinson CM. The cognitive effects of fat embolus syndrome following an isolated femoral shaft fracture. A case report. J Bone Joint Surg Am. 2007;89(5):1092-6.

18. Manousakis G, Han DY, Backonja M. Cognitive outcome of cerebral fat embolism. J Stroke Cerebrovasc Dis. 2012;21(8):906.e1-3.

19. Cicerone KD, Langenbahn DM, Braden C, Malec JF, Kalmar K, Fraas M, et al. Evidence-based cognitive rehabilitation: updated review of the literature from 2003 through 2008. Arch Phys Med Rehabil. 2011;92(4):519-30.

20. Langhorne $\mathrm{P}, \mathrm{Wu} \mathrm{O}$, Rodgers $\mathrm{H}$, Ashburn A, Bernhardt J. A very early rehabilitation trial after stroke (AVERT): a phase III, multicentre, randomised controlled trial. Health Technol Assess. 2017;21(54):1-120.

21. Scarpino M, Lanzo G, Lolli F, Grippo A. From the diagnosis to the therapeutic management: cerebral fat embolism, a clinical challenge. Int J Gen Med. 2019;12:39-48.

22. Srikanth K, Sundararajan S, Rajasekaran S. Late recovery in cerebral fat embolism. Ind J Orthop. 2014:48(1):100-3.

\section{Publisher's Note}

Springer Nature remains neutral with regard to jurisdictional claims in published maps and institutional affiliations. 